UFIFT-HEP-92-22

July, 1992

\title{
Some Simple Criteria for Gauged R-parity
}

\author{
Stephen P. MARTin ${ }^{\dagger}$ \\ Department of Physics \\ University of Florida \\ Gainesville, FL 32611
}

\begin{abstract}
We catalog some simple conditions which are sufficient to guarantee that R-parity survives as an unbroken gauged discrete subgroup of the continuous gauge symmetry in certain supersymmetric extensions of the standard model.
\end{abstract}

$\bar{\dagger}$ Address after Sept. 1, 1992: Dept. of Physics, Northeastern University, Boston MA 02115 
Low energy $N=1$ supersymmetry has been proposed as a cure for the fine-tuning problem associated with the Higgs scalar boson[1]. However, in the minimal supersymmetric extension of the standard model, proton decay might be expected to occur at an unacceptable rate due to the virtual exchange of the superpartners of the standard model states[2,3]. To see this, we can write all of the renormalizable and gauge-invariant terms which might occur in the superpotential:

$$
\begin{aligned}
& W=W_{1}+W_{2}+W_{3} \\
& W_{1}=\mu H \bar{H}+y_{u} Q \bar{H} u+y_{d} Q H d+y_{e} L H e \\
& W_{2}=\lambda_{1} u d d \\
& W_{3}=\mu^{\prime} L \bar{H}+\lambda_{2} Q L d+\lambda_{3} L L e .
\end{aligned}
$$

[Here $Q$ and $L$ are chiral superfields for the $S U_{2}^{L}$-doublet quarks and leptons; $u, d, e$ are chiral superfields for the $S U_{2}^{L}$-singlet quarks and leptons, and $H, \bar{H}$ are the two $S U_{2}^{L}$ doublet Higgs chiral superfields. Family and gauge indices are suppressed. It is possible to eliminate $\mu^{\prime}$ by a suitable rotation among the superfields $H$ and $L$; but we choose not to do this because in most extensions of the minimal supersymmetric standard model $H$ and $L$ will not have the same quantum numbers.] The terms in $W_{1}$ are just the supersymmetric versions of the usual standard model Yukawa couplings and Higgs mass, and they conserve baryon number $(B)$ and lepton number $(L)$. However, $W_{2}$ violates $B$ by one unit and $W_{3}$ violates $L$ by one unit. To prevent the proton from decaying in short order, either $\left(\lambda_{1}\right)$ or $\left(\mu^{\prime}, \lambda_{2}, \lambda_{3}\right)$ must be very small[4].

The simplest way to save the proton and avoid other phenomenological disasters is to just banish all of the terms occuring in $W_{2}$ and $W_{3}$ by means of a discrete $Z_{2}$ symmetry known as R-parity[2,3]. All of the standard model states are taken to be even under R-parity and their superpartners are taken to be odd. All interactions are required to have even R-parity. This means that particles with odd R-parity are always produced in pairs, and that the lightest particle with odd R-parity must be stable. At the level of the chiral superfields, this may be implemented by assigning $R_{p}=-1$ to $Q, L, u, d, e$ and $R_{p}=+1$ to $H, \bar{H}$. (This $R_{p}$ is trivially related to R-parity by a factor of -1 for fermions 
and is usually called matter parity.) Then the terms in $W_{2}$ and $W_{3}$ are forbidden because they are $R_{p}$-odd, while the terms in $W_{1}$ are $R_{p}$-even and allowed. The $R_{p}$ symmetry also forbids some $B$ and $L$-violating operators of dimension five and higher. Other discrete symmetries are possible $[5,6,4]$ which allow some terms from either $W_{2}$ or $W_{3}$, as well as different combinations of higher dimension operators violating $B$ and $L$.

At the level of the minimal supersymmetric standard model, the imposition of $R_{p}$, or any other discrete symmetry designed to protect the proton from decay, appears to be quite ad hoc. This is no longer necessarily true if one considers extensions of the minimal theory in which the standard model gauge group is embedded in a larger gauge symmetry, even if the additional gauge symmetry is broken. Indeed, $R_{p}$ actually follows automatically in certain theories with gauged $B-L$ (e.g. some supersymmetric grand unified theories), and moreover can survive the spontaneous breakdown of the continuous gauge invariance to the standard model gauge group. This will occur if certain conditions, which seem to us to be surprisingly mild, are met by the order parameters of the theory. For some reason, this seems to have been underemphasized in the literature. In this paper, we will catalog some of the simple criteria which are sufficient to guarantee that $R_{p}$ is an unbroken discrete gauge symmetry for various choices of the gauge group, by classifying the possible group transformation properties of the order parameters of the theory as "safe" or "unsafe" for $R_{p}$. For our purposes, it is most convenient to note that for each chiral superfield,

$$
R_{p}=(-1)^{3(B-L)}
$$

This strongly suggests that we obtain gauged $R_{p}$ as the discrete remnant of a gauged $U_{1}^{B-L}$. In fact, with the $U_{1}^{B-L}$ assignments $Q \sim 1 / 3 ; L \sim-1 ; u, d \sim-1 / 3 ; e \sim 1$; and $H, \bar{H} \sim 0$, it is clear that unbroken $U_{1}^{B-L}$ forbids each of the terms in $W_{2}$ and $W_{3}$. To guarantee that $R_{p}$ should remain unbroken even after $U_{1}^{B-L}$ is broken, it is necessary and sufficient to require that all Higgs vacuum expectation values (or other order parameters) carry $3(B-L)$ charges which are even integers[7,6]. Following the general arguments of Krauss and Wilczek[8], $U_{1}^{B-L}$ then breaks down to a gauged $Z_{2}$ subgroup which, in view of (5), is nothing other than $R_{p}$. Unlike a global symmetry, such a gauged discrete symmetry 
will be respected by Planck-scale physics[8].

The most natural setting for gauged $U_{1}^{B-L}$ occurs in the Pati-Salam unification of color and lepton number: $S U_{4}^{P S} \supset S U_{3}^{c} \times U_{1}^{B-L}$. Under the gauge group $S U_{4}^{P S} \times S U_{2}^{L} \times$ $U_{1}^{R}$, the standard model quark and lepton superfields transform as $Q, L \sim(\mathbf{4}, \mathbf{2}, 0)$ and $d, e \sim(\overline{\mathbf{4}}, \mathbf{1}, 1 / 2)$ and $u, N \sim(\overline{\mathbf{4}}, \mathbf{1},-1 / 2)$. [Here $N$ is the superfield for a neutrino which transforms as a singlet under the standard model gauge group.] With unbroken $S U_{4}^{P S}$, the couplings $\lambda_{1}, \lambda_{2}$, and $\lambda_{3}$ clearly vanish by gauge invariance, since the $S U_{4}^{P S}$ direct products $\overline{\mathbf{4}} \times \overline{\mathbf{4}} \times \overline{\mathbf{4}}$ and $\mathbf{4} \times \mathbf{4} \times \overline{\mathbf{4}}$ contain no singlets[9]. Also, gauge invariance of the allowed Yukawa couplings in $W_{1}$ requires that $H$ transforms as a linear combination of $(\mathbf{1}, \mathbf{2},-1 / 2)$ and the color singlet part of $(\mathbf{1 5}, \mathbf{2},-1 / 2)$, and that $\bar{H}$ transforms as a linear combination of $(\mathbf{1}, \mathbf{2}, 1 / 2)$ and the color singlet part of $(\mathbf{1 5}, \mathbf{2}, 1 / 2)$. It then follows that $\mu^{\prime}$ vanishes as well, because it is not allowed by $S U_{4}^{P S}$. So unbroken $S U_{4}^{P S}$ prohibits the same terms in $W_{2}$ and $W_{3}$ that $R_{p}$ does. This is hardly a surprise, since $R_{p}$ is a discrete subgroup of $U_{1}^{B-L}$ which is contained in $S U_{4}^{P S}$.

Of course, $S U_{4}^{P S}$ and $U_{1}^{B-L}$ must be broken if we are to obtain the standard model gauge group. To avoid breaking $R_{p}$ in the process, it is necessary and sufficient that all of the order parameters have even $S U_{4}^{P S}$ quadrality, since we observe that

$$
S U_{4}^{P S} \text { quadrality }=3(B-L) \quad[\bmod 4]
$$

Note that the standard model Higgs superfields $H$ and $\bar{H}$ have zero $S U_{4}^{P S}$ quadrality and thus do not break $R_{p}$ when they acquire vacuum expectation values. Since the order parameters must also be color singlets, we find that they may transform under $S U_{4}^{P S}$ as $1,10,15,35 \ldots$ and their conjugates, which we refer to as "safe" reps. The order parameters should not occur in "unsafe" reps $\mathbf{4}, \mathbf{2 0}^{\prime \prime}, \mathbf{3 6} \ldots$ if we want $R_{p}$ to survive. In particular, the $S U_{2}^{L}$-singlet order parameters may occur in the "safe" reps $(\mathbf{1}, \mathbf{1}, 0)$; $(\mathbf{1 0}, \mathbf{1}, 1) ;(\mathbf{1 5}, \mathbf{1}, 0) ;(\mathbf{3 5}, \mathbf{1}, 2) \ldots$ of $S U_{4}^{P S} \times S U_{2}^{L} \times U_{1}^{R}$ and their conjugates, but not in the "unsafe" reps $(\mathbf{4}, \mathbf{1}, 1 / 2) ;\left(\mathbf{2 0}^{\prime \prime}, \mathbf{1},-3 / 2\right) ; \ldots$ and their conjugates. As long as we arrange for the theory to only have order parameters corresponding to "safe" reps, then $R_{p}$ remains unbroken. 
Actually, $3(B-L)$ is always an integer multiple of 6 for safe order parameters in $S U_{4}^{P S}$, since they must also be color singlets. Thus the surviving discrete subgroup of $U_{1}^{B-L}$ is a $Z_{6}$; however, a $Z_{3}$ subgroup of this is just the discrete center of $S U_{3}^{c}$, which is already taken into account. So the remaining $Z_{2}=R_{p}$ is what really counts. Also note that if all order parameters in the theory had zero $S U_{4}^{P S}$ quadrality, then because of (6) we would be left with a $Z_{4}$ which contains $R_{p}$ as a subgroup and eliminates certain operators of dimension $\geq 5$ which are allowed by $R_{p}$. However, such a situation is very unlikely, since obtaining a realistic neutrino mass spectrum via the seesaw mechanism[10] requires a Majorana mass term for $N$, which in turn requires an order parameter with quadrality 2.

The grand unified theory based on $S O_{10}$ contains $S U_{4}^{P S}$ as a subgroup, and so we may expect to obtain a nice criterion for this case also. The standard model quark and lepton superfields all transform as components of the 16-dimensional spinor rep of $S O_{10}$ : $Q, L, d, e, u, N \sim \mathbf{1 6}$. Now the absence of the couplings $\lambda_{1}, \lambda_{2}$, and $\lambda_{3}$ follows in the language of unbroken $S O_{10}$ from the group theory fact $\mathbf{1 6} \times \mathbf{1 6} \times \mathbf{1 6} \not \supset \mathbf{1}$. Furthermore, since $\mathbf{1 6} \times \mathbf{1 6}=\mathbf{1 0}_{S}+\mathbf{1 2 0}_{A}+\mathbf{1 2 6}_{S}$, it must be that $H$ and $\bar{H}$ are each linear combinations of appropriate components of $\mathbf{1 0}, \overline{\mathbf{1 2 6}}$, and $\mathbf{1 2 0}$ (which couples families antisymmetrically) in order to allow the Yukawa couplings in $W_{1}$. Then from $\mathbf{1 6} \times \mathbf{1 0} \not \supset \mathbf{1}, \mathbf{1 6} \times \mathbf{1 2 0} \not \supset \mathbf{1}$, and $\mathbf{1 6} \times \overline{\mathbf{1 2 6}} \not \supset \mathbf{1}$ it follows that $\mu^{\prime}=0$ also for unbroken $S O_{10}$.

What happens after $S O_{10}$ is broken? The survival or non-survival of $R_{p}$ just depends on the $\mathrm{SO}_{10}$ transformation properties of the order parameters. The relevant property of the $S O_{10}$ reps is the "congruency class" [9] which is defined mod 4 . In fact, we find

$$
S O_{10} \text { congruency class }=3(B-L) \quad[\bmod 2],
$$

so that "safe" reps for order parameters in $S O_{10}$ are just those with congruency class 0 or 2. The safe reps are $\mathbf{1 0}, \mathbf{4 5}, \mathbf{5 4}, \mathbf{1 2 0}, \mathbf{1 2 6}, \mathbf{2 1 0}, \mathbf{2 1 0}, \mathbf{3 2 0} \ldots$ and their conjugates. The "unsafe" reps are 16, 144, $560 \ldots$ and their conjugates. As long as only order parameters corresponding to even $S O_{10}$ congruency classes are used to break $S O_{10}$, the resulting low energy gauge group will inevitably include an unbroken $R_{p}$. The prospect of removing the unsafe reps for Higgs vacuum expectation values from the model builder's palette seems 
unlamentable; for example[11], $S O_{10}$ can be broken down to $S U_{3}^{c} \times S U_{2}^{L} \times U_{1}^{Y} \times R_{p}$ with realistic Yukawa couplings and then to $S U_{3}^{c} \times U_{1}^{E M} \times R_{p}$, using only order parameters in the reps 10, 54 and $\overline{\mathbf{1 2 6}}$. Order parameters in unsafe reps are incapable of giving any masses to the standard model states (or $N$ ) anyway. Note that the safe reps of $S U_{4}^{P S}$ are precisely the ones which are embedded in safe reps of $S O_{10}$, since $S U_{4}^{P S}$ quadrality $=S O_{10}$ congruency class $[\bmod 2]$.

(While chiral superfields in large reps may ruin the asymptotic freedom of the unified gauge coupling, this need not concern us. The order parameters associated with the large reps may find their place only in a phenomenological description, and may not actually correspond to vacuum expectation values for fundamental fields. Also, a Landau singularity in the unified gauge coupling is presumeably irrelevant if it occurs at a distance scale shorter than the Planck length.)

Note that gauged $U_{1}^{B-L}$ does not occur in a pure $S U_{5}$ grand unified theory. In fact, with the standard $S U_{5}$ assignments $L, d \sim \overline{\mathbf{5}}$ and $Q, u, e \sim \mathbf{1 0}$, it is clear that unbroken $S U_{5}$ allows $\lambda_{1}, \lambda_{2}$ and $\lambda_{3}$ equally, by looking at the standard model content of the $S U_{5}$ fact $\overline{\mathbf{5}} \times \overline{\mathbf{5}} \times \mathbf{1 0} \supset \mathbf{1}$. Furthermore, $H$ consists of some $\overline{\mathbf{5}}$ and some $\overline{\mathbf{4 5}}$ and $\bar{H}$ consists of some 5 and some 45; so $\mu^{\prime}$ is also certainly allowed. There is no reason for any of these couplings to vanish in pure $S U_{5}$, in sharp contrast to our other examples.

The case of supersymmetric "flipped" $S U_{5}[12]$ is quite different from pure $S U_{5}$ since it contains gauged $U_{1}^{B-L}$. Under the gauge group $S U_{5} \times U_{1}^{f}$, the standard model fermions transform as $Q, d, N \sim(\mathbf{1 0}, 1) ; L, u \sim(\overline{\mathbf{5}},-3) ;$ and $e \sim(\mathbf{1}, 5)$. Naturally, $W_{2}$ and $W_{3}$ are absent as long as $S U_{5} \times U_{1}^{f}$ is unbroken, which can be seen as a consequence of the $U_{1}^{B-L}$ subgroup. When $S U_{5} \times U_{1}^{f}$ breaks, $R_{p}$ survives if the order parameters transform as components of certain "safe" reps of $S U_{5} \times U_{1}^{f}$. Since $U_{1}^{f}$ charge $=3(B-L)$ mod 2 , the safe reps are just those which have even integer $U_{1}^{f}$ charges in our normalization. These include $(\mathbf{5},-2)$ and $(\overline{\mathbf{5}}, 2)$; the unsafe reps include $(\mathbf{5}, 3)$ and $(\mathbf{1 0}, 1)$ and their conjugates. One of the selling points of flipped $S U_{5}$ is that the spontaneous symmetry breaking can be accomplished using only Higgs fields in reps no larger than the (10,1). However, this 
cannot be accomplished if one insists on using only Higgs fields which are safe for $R_{p}$. If unbroken $R_{p}$ exists in such models, it must come from an additional structure (e.g. superstring theory).

The grand unified theory $E_{6}$ also contains $U_{1}^{B-L}$ as a subgroup. However, reps of $E_{6}$ cannot be classified as safe or unsafe for $R_{p}$, because each irreducible rep contains components with both even and odd values of $3(B-L)$. Since $R_{p}$ is an abelian discrete subgroup, it will suffice for us to classify superfields and possible order parameters in $E_{6}$ according to their transformation properties under the subgroup $S U_{3}^{c} \times S U_{2}^{L} \times U_{1}^{Y} \times U_{1}^{B-L} \times U_{1}^{X}$. (It does not concern us whether this subgroup is actually the unbroken gauge group at any particular stage of symmetry breaking.) The $\mathbf{2 7}$ of $E_{6}$ transforms under this sub$\operatorname{group}$ as $(\mathbf{3}, \mathbf{2}, 1 / 6,1 / 3,1)+(\mathbf{1}, \mathbf{2},-1 / 2,-1,3)+(\overline{\mathbf{3}}, \mathbf{1},-2 / 3,-1 / 3,2)+(\overline{\mathbf{3}}, \mathbf{1}, 1 / 3,-1 / 3,2)+$ $(\mathbf{1}, \mathbf{1}, 1,1,0)+(\mathbf{1}, \mathbf{1}, 0,1,0)+(\overline{\mathbf{3}}, \mathbf{1}, 1 / 3,2 / 3,-4)+(\mathbf{3}, \mathbf{1},-1 / 3,-2 / 3,-2)+(\mathbf{1}, \mathbf{2}, 1 / 2,0,-3)+$ $(\mathbf{1}, \mathbf{2},-1 / 2,0,-3)+(\mathbf{1}, \mathbf{1}, 0,0,6)$. This defines $U_{1}^{X}$, which may be thought of as the extra $U_{1}$ which occurs in $E_{6}$ but not $S O_{10}$. The first five terms may be identified with $Q$, $L, u, d$, and $e$ respectively. (We assume that each of the three standard model families are embedded in the $\mathbf{2 7}$ in the same way.) It then follows that $H$ and $\bar{H}$ transform as $(\mathbf{1}, \mathbf{2},-1 / 2,0,-3)$ and $(\mathbf{1}, \mathbf{2}, 1 / 2,0,-3)$. With these assignments, the Yukawa terms in $W_{1}$ all transform as $(\mathbf{1}, \mathbf{1}, 0,0,0)$ and the terms $u d d, Q L d$ and $L L e$ in $W_{2}$ and $W_{3}$ each transform as $(\mathbf{1}, \mathbf{1}, 0,-1,6)$. The term $L \bar{H}$ in $W_{3}$ transforms as $(\mathbf{1}, \mathbf{1}, 0,-1,0)$. Note that an order parameter $(\mathbf{1}, \mathbf{1}, 0,0,6)$ is necessary so that the non-standard-model particles $(\overline{\mathbf{3}}, \mathbf{1}, 1 / 3,2 / 3,-4)+(\mathbf{3}, \mathbf{1},-1 / 3,-2 / 3,-2)$ can get masses, and to allow $H \bar{H}$ in $W_{1}$. We now classify as safe or unsafe the possible $\Delta I=0$ and $\Delta I=1 / 2$ order parameters which occur in the smallest few reps of $E_{6}$, namely the $\mathbf{2 7}, \mathbf{7 8}, \mathbf{3 5 1}$, and $\mathbf{3 5 1}$. (Safe and unsafe reps in $\overline{\mathbf{2 7}}, \overline{\mathbf{3 5 1}}$ and $\overline{\mathbf{3 5 1 ^ { \prime }}}$ can be found by conjugating the ones below.)

The $\Delta I=0$ order parameters for $E_{6}$ which are safe for unbroken $R_{p}$ are: $(\mathbf{1}, \mathbf{1}, 0,0,0)$, which occurs three times in the $\mathbf{7 8}$ of $E_{6} ;(\mathbf{1}, \mathbf{1}, 0,0,-12)$, which occurs once in the $\mathbf{3 5 1}^{\prime}$; $(\mathbf{1}, \mathbf{1}, 0,0,6)$, which occurs once in the $\mathbf{2 7}$, twice in the $\mathbf{3 5 1}$, and once in the $\mathbf{3 5 1}$; and $(\mathbf{1}, \mathbf{1}, 0,-2,0)$, which occurs once in the $\mathbf{3 5 1} \mathbf{1}^{\prime}$. The unsafe $\Delta I=0$ order parameters for $E_{6}$ are: $(\mathbf{1}, \mathbf{1}, 0,-1,6)$ and $(\mathbf{1}, \mathbf{1}, 0,1,-6)$, which each occur once in the $\mathbf{7 8} ;(\mathbf{1}, \mathbf{1}, 0,1,0)$, 
which occurs once in the $\mathbf{2 7}$, twice in the $\mathbf{3 5 1}$, and once in the $\mathbf{3 5 1} \mathbf{1}^{\prime}$; and $(\mathbf{1}, \mathbf{1}, 0,-1,-6)$, which occurs once in the $\mathbf{3 5 1}$ and once in the $\mathbf{3 5 1} 1^{\prime}$.

The safe $\Delta I=1 / 2$ order parameters for $E_{6}$ transform according to: $(\mathbf{1}, \mathbf{2}, 1 / 2,0,-3)$ and $(\mathbf{1}, \mathbf{2},-1 / 2,0,-3)$, which each occur once in the $\mathbf{2 7}$, three times in the $\mathbf{3 5 1}$ and twice in the $\mathbf{3 5 1} \mathbf{1}^{\prime}$. The unsafe $\Delta I=1 / 2$ reps include $(\mathbf{1}, \mathbf{2}, 1 / 2,1,3)$ and $(\mathbf{1}, \mathbf{2},-1 / 2,-1,-3)$ which each occur once in the $\mathbf{7 8} ;(\mathbf{1}, \mathbf{2},-1 / 2,-1,3)$ which occurs once in the $\mathbf{2 7}$, three times in the $\mathbf{3 5 1}$, and twice in the $\mathbf{3 5 1} \mathbf{1}^{\prime} ;(\mathbf{1}, \mathbf{2}, 1 / 2,1,-9),(\mathbf{1}, \mathbf{2}, 1 / 2,-1,3)$ and $(\mathbf{1}, \mathbf{2},-3 / 2,-1,3)$ which each occur once in the $\mathbf{3 5 1}$ and once in the $\mathbf{3 5 1 ^ { \prime }}$.

Now, using safe $\Delta I=0$ order parameters listed above for $\mathbf{2 7}, \mathbf{3 5 1}$ and $\mathbf{3 5 1}^{\prime}, E_{6}$ can be broken down to $S U_{3}^{c} \times S U_{2}^{L} \times U_{1}^{Y} \times R_{p}$ and the states other than $Q, L, u, d, e$ in the $\mathbf{2 7}$ are all eligible to obtain large masses. Also, safe order parameters for $(\mathbf{1}, \mathbf{2}, 1 / 2,0,-3)$ and $(\mathbf{1}, \mathbf{2},-1 / 2,0,-3)$ provide for standard model masses. So, the statement for $E_{6}$ is that the safe reps for order parameters correspond to those which are necessary anyway to break $E_{6}$ to the standard model with the correct mass spectrum. Order parameters in the unsafe reps are not needed for anything from this point of view, although demanding their absence would eliminate some otherwise attractive symmetry breaking patterns.

Dimension-five operators which violate $B$ and $L$ can also contribute to proton decay. The only such operators consistent with supersymmetry and the standard model gauge group which are not already forbidden by $R_{p}$ are $[u u d e]_{F},[Q Q Q L]_{F}$ and $[L L \bar{H} \bar{H}]_{F}$. The first two of these terms are also allowed by any gauge invariance contained in $S O_{10}$, in view of the standard model content of the group theory fact $16 \times \mathbf{1 6} \times \mathbf{1 6} \times \mathbf{1 6} \supset \mathbf{1}+\mathbf{1}$. However, all three terms (and $H \bar{H}$ in $W_{1}$ ) are prohibited by unbroken $E_{6}$ because of $\mathbf{2 7} \times \mathbf{2 7} \times \mathbf{2 7} \times$ $\mathbf{2 7} \not \supset \mathbf{1}$, etc.; this is a simple consequence of $E_{6}$ triality[9], since $Q, L, u, d, e, H, \bar{H}$ all must have triality 1 . Or, it may be viewed as a consequence of $U_{1}^{X}$ and $U_{1}^{B-L}$ conservation. The fact that these terms are forbidden by an unbroken $E_{6}$ gauge group may provide a means of suppressing them even more heavily than naive expectation in the low energy theory, although the extent to which this is true is model dependent. The order parameter $(\mathbf{1}, \mathbf{1}, 0,0,12)$ breaks $U_{1}^{X}$ to a $Z_{12}$ without allowing the aforementioned terms. An order 
parameter $(\mathbf{1}, \mathbf{1}, 0,0,6)$, which is presumeably necessary as noted earlier, will further break $U_{1}^{X}$ to a $Z_{6}$ and allow $[u u d e]_{F},[Q Q Q L]_{F}$ and $[H \bar{H}]_{F}$. This $Z_{6}$ is nothing new, because it is just a subgroup of $Z_{3}^{c} \times Z_{2}^{L}$, where $Z_{3}^{c}$ is the center of $S U_{3}^{c}$ and $Z_{2}^{L}$ is the center of $S U_{2}^{L}$. The order parameter $(\mathbf{1}, \mathbf{1}, 0,-2,0)$ breaks $U_{1}^{B-L}$ to $R_{p}$ and allows $[L L \bar{H} \bar{H}]_{F}$.

If $R_{p}$-unsafe order parameters do occur in theories with gauged $U_{1}^{B-L}$, then $R_{p}$ is spontaneously broken. This is not necessarily a disaster if the mass scales of the unsafe order parameters are sufficiently small compared to the unification scale. One popular example which has been explored in the literature[13] involves spontaneous $R_{p}$ breaking due to an expectation value for the scalar partner of a neutrino which transforms in the unsafe rep $(\mathbf{1}, \mathbf{1}, 0,1,0) \subset \mathbf{2 7}$ of $S U_{3}^{c} \times S U_{2}^{L} \times U_{1}^{Y} \times U_{1}^{B-L} \times U_{1}^{X} \subset E_{6}$, or the unsafe rep $\mathbf{1 6}$ of $S O_{10}$. There are, of course, other possible examples (of problematical phenomenological viability) for spontaneously broken $R_{p}$ if other unsafe reps listed above obtain vacuum expectation values. The dominant contributions to $B$ and $L$-violating terms in the low energy effective superpotential come from tree graphs with unsafe order parameters on external legs. Note that explicit $R_{p}$ breaking is really a contradiction in terms for any model with gauged $U_{1}^{B-L}$, because of (5); it must be either exact or spontaneously broken.

In superstring-inspired models based on remnants of $E_{6}$, the existence of $R_{p}$ depends on e.g. the properties of the six-dimensional compactified manifold. There are a bewildering plethora of possibilities for the vacuum, some of which respect $R_{p}$ or other generalized matter parities, and some of which do not. Note that it is not possible to break the $U_{1}^{B-L}$ subgroup to $R_{p}$ in these models after compactification, because the necessary safe order parameter resides in the $\mathbf{3 5 1 ^ { \prime }}$ (or larger) rep of $E_{6}$, and not in the $\mathbf{2 7}, \overline{\mathbf{2 7}}$, or singlet reps which are available for chiral superfields in the perturbative field theory limit of superstring theory. In such models it is not possible to understand in detail "why" unbroken $R_{p}$ should exist without understanding the compactification mechanism. In this paper we have considered instead criteria which can be understood using only an effective $N=1$ supersymmetric model. These criteria are reassuringly not too restrictive, and are especially crisp in the languages of $U_{1}^{B-L}, S U_{4}^{P S}$ and $S O_{10}$. Hopefully, R-parity appears less ad hoc in this light. 
I am grateful to Pierre Ramond for helpful comments. This work was supported in part by the Institute for Fundamental Theory and by DOE contract DE-FG05-86-ER40272.

\section{References}

1. For reviews, see H. P. Nilles, Phys. Reports 110, 1, (1984) and H. E. Haber and G. L. Kane, Phys. Reports 117, 75, (1985).

2. G. Farrar, P. Fayet, Phys. Lett. B76, 575, (1978); S. Dimopolous and H. Georgi, Nucl. Phys. B193, 150, (1981).

3. S. Weinberg, Phys. Rev. D 26, 287, (1982); N. Sakai and T. Yanagida, Nucl. Phys. B197, 83, (1982).

4. For precise constraints, see references [1], [2], and [3] and F. Zwirner, Phys. Lett. B132, 103, (1983); L. J. Hall and M. Suzuki, Nucl. Phys. B231, 419, (1984); I. H. Lee, Nucl. Phys. B246, 120, (1984); S. Dawson, Nucl. Phys. B261, 297, (1985); R. Barbieri and A. Masiero, Nucl. Phys. B267, 679, (1986); S. Dimopolous, L. J. Hall, Phys. Lett. B207, 210, (1987); V. Barger, G. F. Giudice, and T. Han, Phys. Rev. D 40, 2987, (1989); S. Dimopolous, R. Esmailzadeh, L. J. Hall, J.-P. Merlo, and G. D. Starkman, Phys. Rev. D 41, 2099, (1990); R. Barbieri, D. E. Brahm, L. J. Hall and S. D. H. Hsu, Phys. Lett. B238, 86, (1990); B. A. Campbell, S. Davidson, J. Ellis and K. A. Olive, Phys. Lett. B256, 457, (1991); H. Dreiner and G. G. Ross, Nucl. Phys. B365, 597, (1991).

5. M. C. Bento, L. Hall and G. G. Ross, Nucl. Phys. B292, 400, (1987).

6. L. E. Ibáñez and G. G. Ross, Phys. Lett. B260, 291, (1991); Nucl. Phys. B368, 3, (1992).

7. A. Font, L. E. Ibáñez and F. Quevedo, Phys. Lett. B228, 79, (1989).

8. L. Krauss and F. Wilczek, Phys. Rev. Lett. 62, 1221, (1989). 
9. All group theory conventions and facts used in this paper may be found in R. Slansky, Phys. Reports 79, 1, (1981).

10. M. Gell-Mann, P. Ramond, and R. Slansky, in Sanibel Talk, CALT-68-709, Feb 1979, and in Supergravity, (North Holland, Amsterdam, 1979; T. Yanagida, in Proc. of the Workshop on Unified Theories and Baryon Number in the Universe, Tsukuba, Japan, 1979, edited by A. Sawada and A. Sugamoto (KEK Report No. 79-18, Tsukuba, 1979).

11. J. A. Harvey, P. Ramond, and D. B. Reiss, Phys. Lett. B92, 309, (1980); Nucl. Phys. B199, 223, (1982).

12. A. De Rújula, H. Georgi, and S. L. Glashow, Phys. Rev. Lett. 45, 413, (1980); S. M. Barr, Phys. Lett. B112, 219, (1982); I. Antoniadis, J. Ellis, J. S. Hagelin, and D. V. Nanopoulos, Phys. Lett. B199, 231, (1987).

13. C. S. Aulakh and R. N. Mohapatra, Phys. Lett. B119, 136, (1982); J. Ellis, G. Gelmini, C. Jarlskog, G. G. Ross, and J. W. F. Valle, Phys. Lett. B150, 142, (1985); G. G. Ross and J. W. F. Valle, Phys. Lett. B151, 325, (1985); B. Gato, J. Leon, J. PerezMercader, and M. Quiros, Nucl. Phys. B260, 203, (1985); A. Masiero and J. W. F. Valle, Phys. Lett. B251, 273, (1990). 